\title{
Research and Development of High Performance Soft Magnetic Thin Films
}

\author{
Tetsuya OSAKA, ${ }^{*}$ Toshiyuki MOMMA, and Tokihiko YoKOSHIMA
}

Department of Applied Chemistry, School of Science and Engineering; Kagami Memorial Laboratory for Materials Science and Technology, Waseda University (3-4-1 Okubo, Shinjuku-ku, Tokyo 169-8555, Japan)

Received June 1, 1999 ; Accepted June 9, 1999

\begin{abstract}
Soft magnetic films for head core materials mainly electrodeposited CoNiFe based films developed by us were introduced. Electrodeposited CoNiFe thin film has high saturation magnetic flux density $\left(B_{s}\right)$ and low coercivity. Magnetic properties except for $B_{s}$ and corrosion properties of these films were depended on very small amount of inclusion such as $\mathrm{S}$ and $\mathrm{H}$ elements. The increase in resistivity $(\rho)$ on the base of this high $B_{s} \mathrm{CoNiFe}$ thin film was developed by controlling very small amount of $\mathrm{C}$ inclusion. Finally, application of the film to new type of magnetic recording head was reported.
\end{abstract}

Key Words : Electrodeposition, Soft Magnetic Film, High $B_{s}$ Film, High $\rho$ Film

\section{Introduction}

In the field of application of highly functional thin films, it is required to regulate the properties more critically. In the case of magnetic films, a saturation magnetic flux density $\left(B_{s}\right)$ greater than 1.0 tesla $(\mathrm{T})$ is required for soft magnetic materials being used for miniaturized magnetic sensors, step motors, and magnetic recording heads. For these applications, many different soft magnetic films with high $B_{s}$ have been investigated extensively. Examples are electroplated $\mathrm{CoFe}$ with $B_{s}=1.8-1.9 \mathrm{~T},{ }^{1)}$ electroplated $\mathrm{CoNiFe}$ with $B_{s}=1.6-1.8 \mathrm{~T},{ }^{2-4)}$ and sputtered Fe-based nano-crystalline alloys such as $\mathrm{FeN}$ based films with $B_{s}=1.4-1.9 T^{5,6)}$ However, the process of sputtering is not suitable for fabricating magnetic circuits with high aspect ratios. Thus, an electrochemical process is preferred.

Electrochemical methods have been used for practical production of conventional permalloy $\left(\mathrm{Ni}_{80} \mathrm{Fe}_{20}\right.$ (at.\%) ) films. Especially, electroplated permalloy has been playing a key role in the technology of manufacturing magnetic recording heads and micromagnetic actuators. It is based on the technology of fabricating magnetic wire memories prevalent as early as in the 1950-60's. Many permalloy plating baths used in the industry generally contain organic additive(s) with sulfur functional group(s). The organic additives are used to reduce inner stress of the deposited film to decrease the grain size and to lower the coercivity $\left(H_{c}\right)^{7}{ }^{7}$ Such sulfur-containing additives (SCA) are known to cause the inclusion of sulfur in the film through their surface activities, ${ }^{7)}$ and those additives will hereafter be called SCA. Furthermore, not only the additives, it is known that other operating conditions are important to control the properties of the resulting film. In general, the parameters to plate functional films are so compli- cated to realize the required properties.

In this paper, the investigation of high performance soft magnetic films developed by us is summarized with the study on the mechanism exhibiting the soft magnetic properties.

\section{Results and Discussion}

2. 1 Effect of sulfur inclusions on the magnetic properties for CoNiFe soft magnetic films

Figure 1 shows maps of magnetic properties of CoNiFe films electroplated in three different baths. The baths used to generate Figs. $1 \mathrm{~A}$ and $1 \mathrm{~B}$ contained saccharin $^{8)}$ and thiourea, ${ }^{9,10)}$ respectively, which cause co-deposition of a small amount of sulfur in the films, whereas the bath used for Fig. 1C does not contain such an active additive of SCA. It is seen in Fig. 1A that the region of $B_{s}>1.8 \mathrm{~T}$ is located in the Fe rich and Co rich area. The regions of $B_{s}>1.8 \mathrm{~T}$ in Figs. $1 \mathrm{~B}$ and $1 \mathrm{C}$ are located in almost the same compositional area as in Fig. 1A, which indicates that $B_{s}$ is essentially determined by relative concentrations of the three metallic elements. The lines of zero magnetostriction, $\lambda_{s}=0$, are located in the $\mathrm{Fe}$ poor area. It is important that $\lambda_{s}$ of the film for fabricating the magnetic head core should be low because magnetic field does not produce stress in such films. Furthermore, the region of low $H_{c}$ and the line indicating fcc-bcc phase boundary are shown in the figures. Comparison of these figures with the information provided by Bozorth ${ }^{11)}$ for bulk $\mathrm{CoNiFe}$ alloy indicates that the region of low $H_{c}$, and the lines of $\lambda_{s}=0$ and the fcc-bcc phase boundary are quite different from the films and for the bulk alloy, but their $B_{s}$ values are not. In addition, the aforementioned properties are generally different from those of the films prepared by the other thin film techniques such as sputtering and quenching. It is thus likely that the 
newly developed soft magnetic film can be prepared only by electroplating. In fact, it has already been reported that an electroplated $\mathrm{CoNiFe}$ film with an especially low $H_{c}$ value of $19.8 \mathrm{~A} / \mathrm{m}(0.25 \mathrm{Oe})$ contains small crystallites near the region of fcc-bcc phase boundaries. However, CoNiFe films with $H_{c}<159$ $\mathrm{A} / \mathrm{m}(2 \mathrm{Oe})$ and $B_{s}>1.9 \mathrm{~T}$ and also even soft magnetic films with $H_{c}<159 \mathrm{~A} / \mathrm{m}(2 \mathrm{Oe})$ and $B_{s}>2.0 \mathrm{~T}$, have not been reported thus far. The $H_{c}$ is greatly dependent on the crystal grain size in the entire film. Thus, we made an attempt to find an alternate, more effective additive in place of saccharin for this purpose. It was found that thiourea is an effective additive, which shifts the low $H_{c}$ region into the high $B_{s}$ region as shown in Fig. 1B. With thiourea the lines for $\lambda_{s}=0$ and for the fcc-bcc boundary also shifted toward the Fe-poor compositional region. The film contained a small amount of sulfur, 0.3 to 3.5 at.\%, as determined by energy dispersive spectroscopy (EDS). The inclu-

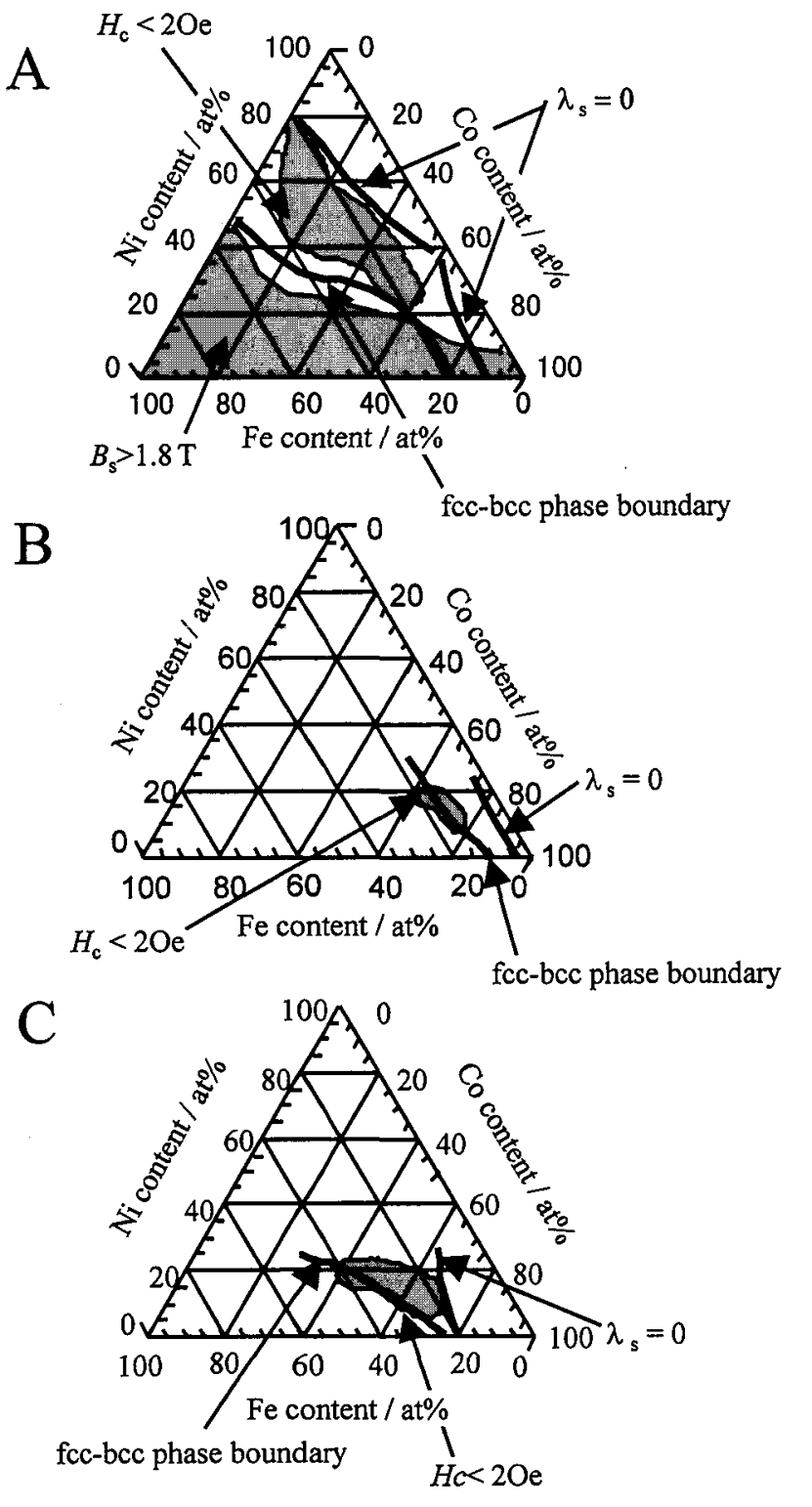

Fig. 1 Magnetic properties and fcc-bcc phase boundary of electrodeposited CoNiFe film from saccharin bath (A), $\mathrm{CoNiFeS}$ film from thiourea bath (B), and High- $B_{s} \mathrm{CoNiFe}$ film from No-SCA bath (C). sion of the larger amount of sulfur from thiourea was found to cause the crystal grains to become smaller, which was confirmed by examination with TEM. These factors are considered to be responsible for the shift of the low $H_{c}$ region into the high $B_{s}$ region. This result demonstrates that selection of a proper additive is one of the important factors in obtaining desired soft magnetic properties. It is of interest to note that both saccharin and thiourea chemisorb on metal surfaces through the sulfur atoms in these compounds. ${ }^{12)}$

On the other hand, for the purpose of comparison, we attempted to shift the line of fcc-bcc phase boundary to a higher $B_{s}$ region using baths of various compounds without such surface-active SCA. As shown in Fig. 1C, which was obtained with one of such baths, a suitable region of high $B_{s}$ with low $H_{c}$ was found without the use of SCA. ${ }^{13-15}$ ) The line of fccbcc mixed phase boundary clearly moved to the left. It is also seen in the figures that the removal of SCA shifts the line of $\lambda_{s}=0$ to a higher Fe compositional region. Thus, we found that a low $H_{c} \mathrm{CoNiFe}$ film with $B_{s}$ as high as 2.0 to $2.1 \mathrm{~T}$ and $\lambda_{s}$ as low as $10^{-6}$ can be prepared without the use of SCA. One of the most suitable films has a composition of $\mathrm{Co}_{65} \mathrm{Ni}_{12} \mathrm{Fe}_{23}$ (at.\%) with $B_{s}=2.1 \mathrm{~T}, H_{c}=95 \mathrm{~A} / \mathrm{m}(1.2 \mathrm{Oe}), \lambda_{s}=1.8 \times$ $10^{-6}$, and $\rho=21 \mu \Omega-\mathrm{cm}$. Figure 2 shows a highresolution transmission electron micrograph (HRTEM) of the high $B_{s} \mathrm{CoNiFe}$ film. It is seen that this film is composed of crystal grains with diameters ranging from 10 to $20 \mathrm{~nm}$. From the result of compositional analysis by Micro-EDX with HR-TEM, it is seen that the compositions were essentially identical at the center of a grain and at a grain boundary, indicating that the high $B_{s}$ CoNiFe film is highly homogeneous in composition.

The influence of the small amount of inclusion and the crystalline structure of the CoNiFe film on the corrosion resistance is also an important factor for the application of the film to the manufacturing process. Figure 3 shows the anodic polarization curves of the films with various content of S inclusion. ${ }^{16)}$ From the figure, it is clear that the corrosion resistivity of CoNiFe film is strongly influenced by the very small amount of $\mathrm{S}$ inclusion. The films deposited with saccharin and thiourea, respectively, such as samples

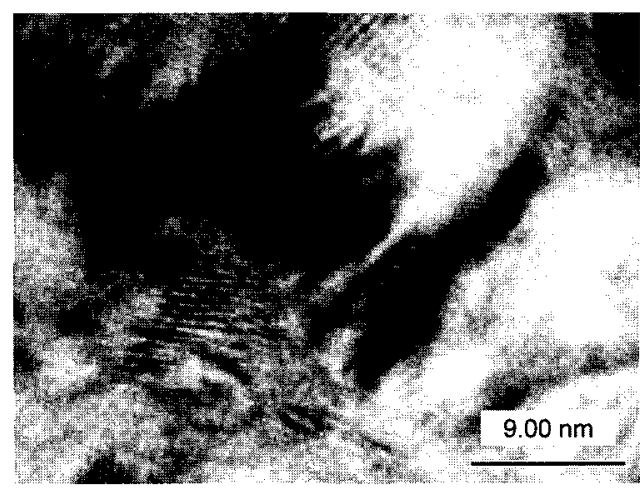

Fig. 2 High-resolution transmission electron micrograph of the electrodeposited High $B_{s} \mathrm{CoNiFe}$ thin film. 


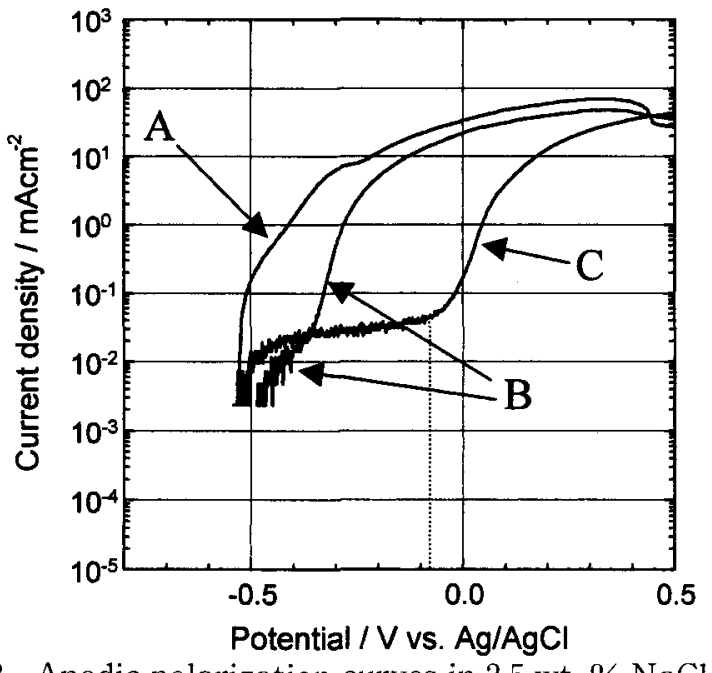

Fig. 3 Anodic polarization curves in $2.5 \mathrm{wt}$. $\% \mathrm{NaCl}$ solution at a scan rate of $10 \mathrm{mV} \mathrm{s}^{-1}$. The electrolyte solution was degassed with $\mathrm{N}_{2}$ bubbling for 5 min. prior to use. Dotted lines show pitting corrosion potentials.

A : CoNiFe thin film from saccharin bath, B:CoNiFeS thin film from thourea bath, $\mathrm{C}: \mathrm{CoNiFe}$ thin film from No-SCA bath.

$\mathrm{A}$ and $\mathrm{B}$, do not show the passivated states, while the film A deposited without these additives, which has quite a few amount of $\mathrm{S}$ impurity, shows the plateau in the curve. By comparing the curves with $\mathrm{A}$ and $\mathrm{B}$, the anodic current for $B$ shows higher value, while the amount of $\mathrm{S}$ indicates the opposite tendency based on the $\mathrm{S}$ influence on the corrosion. This can be explained with the effect of grain boundaries to the corrosion discussed above. From the TEM study, the sample $B$ was found to have the well defined larger grains than the sample $C$. In addition, for the sample A the segregation of $\mathrm{S}$ atom was found. It is well known that corrosion can start easily at the grain boundaries and the segregated region. On the other hand, the sample $\mathrm{C}$ has a large portion of amorphous state, which has high resistivity against the corrosion.

In addition, it is known that the crystalline structure and the grain sizes are strongly influence on the corrosion behavior of metals. Three CoNiFe films containing almost no $\mathrm{S}$ inclusion having $\mathrm{fcc}$, bcc, mixed crystalline of fcc-bcc was examined for the resistivity against the corrosion as shown Fig. 4. Clearly the corrosion potential of sample with mixed crystalline one is higher than both samples of bcc and fcc. The anodic polarization curves for the samples of bcc-fcc and fcc, looked similar. There appears plateau which indicates the passivation state of the sample with the sudden increase of current for the pitting corrosion. There is also a difference between the pitting potentials for mixed one and fcc one. From the pitting potentials, mixed crystalline one was found to have higher resistance against corrosion than fcc one. In the case of bcc structure sample, the current increases with a shoulder without plateau. On the other hand, for the bcc structure sample, there appears no clear passivation current but dissolution current even through the passivation potential area

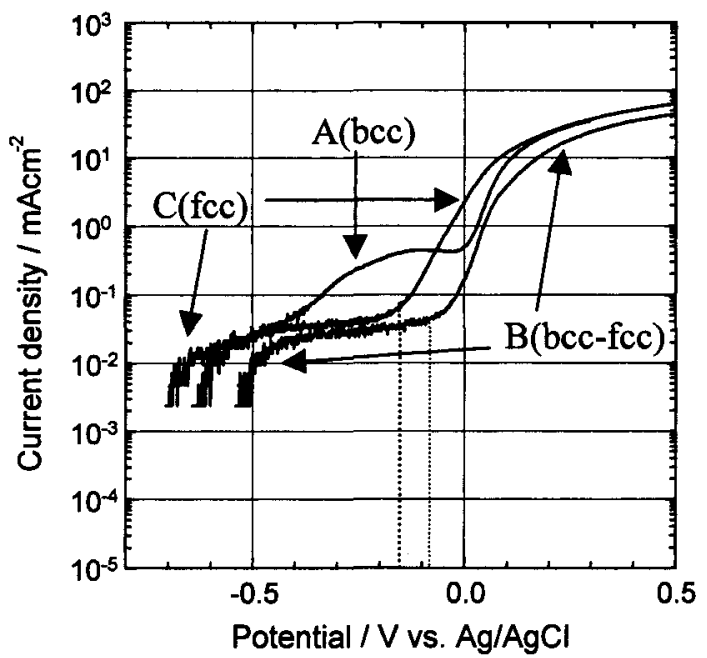

Fig. 4 Anodic polarization curves in $2.5 \mathrm{wt}$. \% $\mathrm{NaCl}$ solution at $10 \mathrm{mV} \mathrm{s}^{-1}$. The electrolyte solution was degassed with $\mathrm{N}_{2}$ bubbling for $5 \mathrm{~min}$. prior to use. Dotted line shows pitting corrosion potential.

A : bcc structure, B : bcc-fcc structure, C : fcc structure.

are seen. It means that the sample of bcc structure has no anticorrosion properties as compared with fcc and bcc-fcc samples.

In conclusion of this session, a few amount of $\mathrm{S}$ inclusion changes the magnetic property and corrosion property of the film drastically by the change in the composition and structure of the film.

\section{2 Effect of $\mathrm{H}_{2}$ evolution on film characteristics} during the alloy deposition of soft magnetic films

In the study for the soft magnetic films deposited without SCA described above, we found that the films prepared with the same elemental composition showed different $H_{c}$ values with changing the operating condition including the bath composition of the film. In the science of the magnetic alloys, the crystalline structure is also the important feature exhibiting the magnetic properties including the coercivity $H_{c}$ with the alloy composition. Beside that in the field of the electrodeposition the selection of operating condition is the powerful way and the only way to control the structural features of the deposits. In order to clarify the relation between the operating condition and the structure of the resulting film, the films of the same composition of $\mathrm{Co}_{62} \mathrm{Ni}_{12} \mathrm{Fe}_{26}$ (at.\%) of no SCA were prepared with a variation of operating condition, especially of bath $\mathrm{pH}$ and current density adjusting the bath composition. ${ }^{17)}$ From surveying the $H_{c}$ values for the $\mathrm{CO}_{62} \mathrm{Ni}_{12} \mathrm{Fe}_{26}$ films, it was found that the $H_{c}$ values of the film showed the tendency of low values when the film was deposited under low current density and / or from the bath of low $\mathrm{pH}$. The results obtained by TEM and XRD revealed that the size of the grains becomes finer with the decrease of $H_{c}$ value of the $\mathrm{Co}_{62} \mathrm{Ni}_{12} \mathrm{Fe}_{26}$ films. With relationship between the operating conditions and the grain size, each step of alloy deposition was considered one by one, with checking the value of $H_{c}$. Figure 5 shows the diagram plotted the $H_{c}$ values of the $\mathrm{Co}_{62} \mathrm{Ni}_{12} \mathrm{Fe}_{26}$ films against the current efficiency 


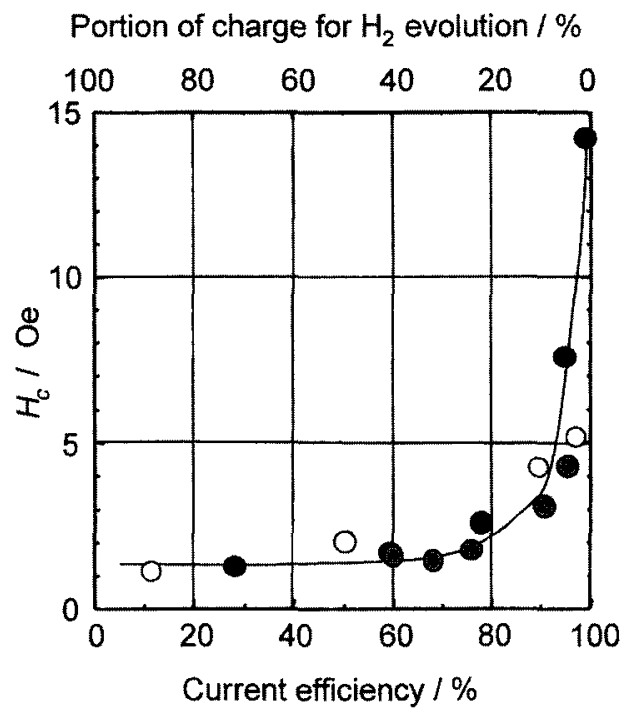

Fig. 5 Relationship between coercivity and current efficiency for electrodeposited $\mathrm{Co}_{62} \mathrm{Ni}_{12} \mathrm{Fe}_{26}$ thin films;

: constant current $\left(=20 \mathrm{~mA} \mathrm{~cm}^{-2}\right)$, : constant $\mathrm{pH}(=$ $2), \bigcirc$ : other operating condition.

during the deposition of the film. It is obvious that there is some correlation between the value of $H_{c}$ and that of current efficiency. That can be discussed with the side-reaction of the electroplating. With the polarization of electrode in the plating bath free of metallic ions, there happens $\mathrm{H}_{2}$ evolution without the other reaction, such as the decomposition of solute in the bath, at the potential applied for the electrodeposition. The results of elemental analysis for no SCA $\mathrm{CoNiFe}$, it is also clear that there seems to be no side-reaction caused by solute except for the $\mathrm{H}_{2}$ evolution. So the reduction of $\mathrm{H}^{+}$into $\mathrm{H}_{2}$ should be the only side-reaction of the electrodeposition of $\mathrm{CO}_{62} \mathrm{Ni}_{12}$ $\mathrm{Fe}_{26}$ without SCA. With the assumption that the rest of the charge passed for the deposition of metal is used for the $\mathrm{H}^{+}$reduction, the charge for the $\mathrm{H}_{2}$ evolution is calculated, as is indicated in Fig. 5. Also the grain size was fine when the film was deposited with the current efficiency under $80 \%$. This fact suggests that circumstances at the interface between the electrolyte and electrode, such as mobility of atom and molecule, phase condition, side reaction, etc., influence strongly on the resulting structure of the film.

In conclusion of this section, it is revealed that the selection of the operating condition for the electrode- position influences the results drastically, and the side reaction during the deposition changes the magnetic property of the film drastically by the change in the structure of the film.

\section{3 Resistivity change of Soft Magnetic Film by C inclusion}

From the viewpoint of data rate of recording, high resistivity $(\rho)$ of the head core materials is requested to decrease the eddy-current loss. The electrodeposited soft magnetic materials with high $\rho$ have not been studied well as compared with high $B_{s}$ materials, while some attempts are made for soft magnetic thin films with high $\rho$ such as electrodeposited NiFe, ${ }^{18}$ $\mathrm{NiFeMo}{ }^{19)}$ or $\mathrm{NiFeP} .{ }^{20)}$ There is no report on soft magnetic materials satisfying both of high $B_{s}$ and high $\rho$ for the writing-head of high-density magnetic recording system of near future.

In order to develop a new soft magnetic thin film with both high $B_{s}$ and high $\rho$, an attempt on increasing the resistivity was investigated on the basis of electrodeposited high $B_{s} \mathrm{CoNiFe}$ thin film. Recently electroless-deposited NiP film with extremely high $\rho$ value of $5000 \mu \Omega-\mathrm{cm}$ from the bath containing a chemical agent having $-\mathrm{NH}_{2}$ groups as the complexing agent, was reported by us. ${ }^{21,22)}$

Based on this result, in order to realize the highly resistive soft magnetic film, we attempted to use the some organic agent having $-\mathrm{NH}_{2}$ groups as the additive to the bath for electrodepotion instead of the complexing agent. Diethylenetriamine (DET) was applied to the bath for the soft magnetic films as an additive. ${ }^{18,20)}$ In the case of $\mathrm{Ni}_{80} \mathrm{Fe}_{20}$ permalloy, $\rho$ value of the film increases from $20 \mu \Omega$-cm to 60 $\mu \Omega-\mathrm{cm}$. It is clear that a small amount of inclusion of $C$ into the soft magnetic film incorporated by the additive of DET makes the resistivity of the film higher. So this method of using DET as the additive to the film deposition bath is revealed to be a possible way to make the film resistance higher.

The electrodeposited $\mathrm{CoNiFe}$ film made with no SCA, which exhibited higher performance as the soft magnetic film than permalloy as described in the former section, was examined for the improvement of resistivity with the DET additive into the deposition bath. ${ }^{23)}$ The optimum properties are listed in Table 1. Figure 6 shows the frequency dependence of permeability of both CoNiFe films with low and high resistivity. Clearly the higher frequency loss is

Table 1 Magnetic and electric properties of electrodeposited high $B_{s} \mathrm{CoNiFe}$ thin films with various $C$ content.

\begin{tabular}{cccc}
\hline \hline Composition & $\mathrm{CO}_{56} \mathrm{Ni}_{13} \mathrm{Fe}_{31}$ & $\left(\mathrm{Co}_{56} \mathrm{Ni}_{13} \mathrm{Fe}_{31}\right)_{99.76} \mathrm{C}_{0.24} *$ & $\left(\mathrm{CO}_{56} \mathrm{Ni}_{13} \mathrm{Fe}_{31}\right)_{99.70} \mathrm{C}_{0.30}{ }^{* *}$ \\
\hline$B_{s} / \mathrm{T}$ & 2.1 & 1.9 & 1.7 \\
$H_{c} / \mathrm{Oe}$ & 1.2 & 2.3 & 6 \\
$\rho / \mu \Omega-\mathrm{cm}$ & 26 & 90 & 130 \\
$\mu /($ at $30 \mathrm{MHz})$ & 550 & 450 & 360 \\
$H_{k} / \mathrm{Oe}$ & 30 & 45 & 45 \\
\hline
\end{tabular}

${ }^{*},{ }^{* *}$ : Films were electrodeposited from the baths with DET 8 and $20 \mathrm{~g} \mathrm{dm}^{-3}$, respectively. 
disappeared with an increase in $\rho$ shown in Fig. 6B. Therefore, it was considered that the increase of $\rho$ value was attributable to change of microstructure and the effect of the impurity scattering because of co-deposition of carbon in the film.

From this study, it is clear that the controlling the small amount of $\mathrm{C}$ in the electrodeposited soft magnetic film was so critical and a powerful tool to realize high performance functional films with desired properties.

\section{4 For next generation fine magnetic recording} head

Figure 7 shows the schematic MR head using high $B_{s} \mathrm{CoNiFe}$ film for writing-head core materials. ${ }^{24}$ The core was fabricated with high $B_{s} \mathrm{CoNiFe}$ film with $0.7 \mu \mathrm{m}$ thick and the thicker permalloy films with $2.3 \mu \mathrm{m}$. When using this new head, the medium higher coercivity such as 7000 Oe is clearly written as reported in ref. 24 . The results indicate the possibility of the next generation recording of $100 \mathrm{Gbit} \mathrm{in}^{-2}$.

Additionally, in over $40 \mathrm{Gbit}^{-2}{ }^{-2}$, the electrolessdeposition method becomes advantageous, because it can be plated without flame plating, and also high step coverage feature. We are still developing various high functional soft magnetic thin films using electroless deposition, ${ }^{25-28)}$ and we are applying this electroless-deposition method to micro-patterning process for next generation fabrication technique. ${ }^{29-32)}$

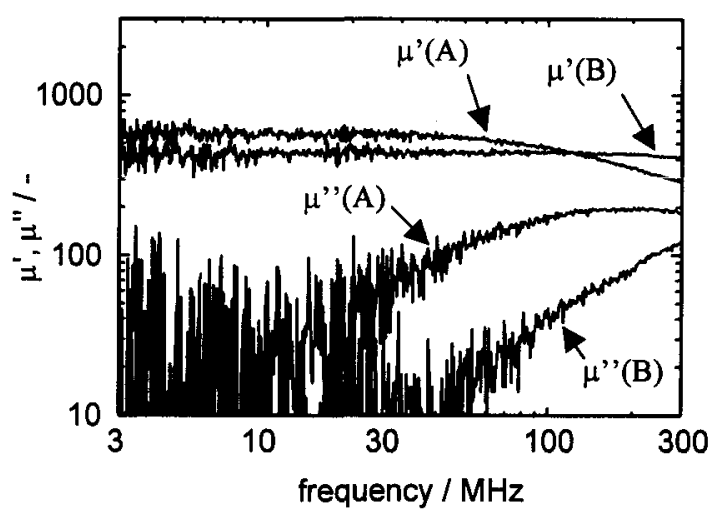

Fig. 6 Relative permeability of CoNiFe thin films ; (A) CoNiFe thin film with $25 \mu \Omega-\mathrm{cm}$, (B) CoNiFe thin film with $90 \mu \Omega$-cm.

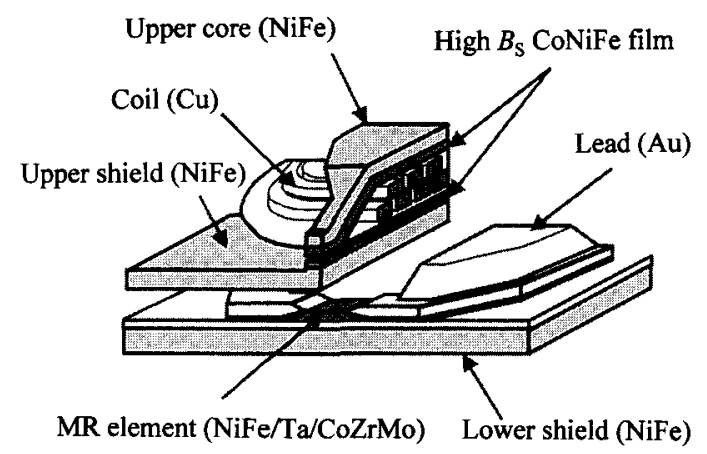

Fig. 7 Schematic view of merged MR head using high $B_{s}$ CoNiFe thin film.

\section{Conclusion}

The magnetic properties of electrodeposited soft magnetic films, mainly CoNiFe films, are summarized in the paper. The electrochemical method has a high potential to make the high performance thin film. Therefore, new soft magnetic materials developed by using the electrodeposition method have high potential to be applied to the high-density magnetic recording head cores for practical use.

\section{References}

1) S. H. Liao, IEEE Trans. Magn., MAG-23, 2981 (1987).

2) N. C. Anderson and R. B. Chesnutt (IBM), U. S. Patent, 4, 661, 216 (1987).

3) Y. Omata, S. Mitani, T. Taniguchi, and S. Nakagawa, J. Magn. Soc. Jpn, 14, 111 (1990) (in Japanese).

4) O. Shinoura, A. Kamijima, and Y. Narumiya, J. Magn. Soc. Jpn, 18, 277 (1994) (in Japanese).

5) N. Ishiwata, C. Wakabayashi, and H. Urai, J. Appl. Phys., 69, 5616 (1991).

6) M. Takahashi, H. Shoji, M. Abe, H. Komaba, and T. Wakiyama, J. Magn. Soc. Jpn, 14, 283 (1990) (in Japanese).

7) R. S. Smith, L. E. Godycki, and J. C. Lloyd, J. Electrochem. Soc., 108, 996 (1961).

8) A. Nakamura, M. Takai, K. Hayashi, and T. Osaka, $J$. Surf. Finsh. Soc. Jpn., 47, 934 (1996) (in Japanese).

9) M. Takai, K. Hayashi, M. Aoyagi, and T. Osaka, $J$. Electrochem. Soc., 144, L203 (1997).

10) M. Takai, K. Hayashi, M. Aoyagi, and T. Osaka, J. Magn. Soc. Jpn., 21-S2, 443 (1997).

11) R. M. Bozorth, Ferromagnetism, D. Van Nostrand Company, Inc., New York, N.Y., p. 160 (1951).

12) T. Osaka, T. Sawaguchi, F. Mizutani, T. Yokoshima, M. Takai, and Y. Okinaka J. Electrochem. Soc., in press.

13) T. Osaka, M. Takai, K. Hayashi, K. Ohashi, M. Saito, and K. Yamada, Nature, 387, 796 (1998).

14) T. Osaka, M. Takai, K. Hayashi, Y. Sogawa, K. Ohashi, Y. Yasue, M. Saito, and K. Yamada, IEEE Trans. Magn., 34, 1632 (1998).

15) K. Ohashi, Y. Yasue, M. Saito, K. Yamada, T. Osaka, M. Takai, and K. Hayashi, IEEE Trans. Magn., 34, 1432 (1998).

16) T. Osaka, M. Takai, Y. Sogawa, T. Momma, K. Ohashi, M. Saito, and K. Yamada, J. Electrochem. Soc., 146, 2092 (1999).

17) Y. Sogawa, S. Mizutani, T. Momma, T. Osaka, M. Saito, K. Ohashi, and K. Yamada, J. Magn. Soc. Jpn., 23, 1405 (1999) (in Japanese).

18) M. Takai, F. Mera, A. Kondo, M. Kaseda, and T. Osaka, J. Surf. Finsh. Soc. Jpn, 49, 292 (1997) (in Japanese).

19) O. Shinoura, Denki Kagaku (presently Electrochemistry), 63, 473 (1995).

20) M. Takai, F. Mera, M. Kaseda, and T. Osaka, J. Magn. Soc. Jpn, 22, 629 (1997) (in Japanese).

21) T. Hatsukawa, T. Higashikawa, T. Osaka, and H. Nakao, J. Surf. Finsh. Soc. Jpn., 47, 779 (1996) (in Japanese).

22) A. Iizuka, T. Higashikawa, and T. Osaka, Proc. of the 3rd Intern. Symp. on Electrochemically Deposited Thin Films, Electrochem Soc., PV96-19, 184 (1996). 
23) T. Yokoshima, M. Kaseda, M. Yamada, T. Nakanishi, T. Momma, and T. Osaka, IEEE Trans. Magn., in press.

24) K. Ohashi, N. Morita, T. Tsuda, and Y. Nonaka, Digests of Intermag '99, FB-04, Korea (1999).

25) M. Takai, K. Kageyama, S. Takefusa, A. Nakamura, and T. Osaka, IEICE Trans. Electron., E78-C, 1530 (1995).

26) T. Osaka, T. Homma, N. Masubuchi, K. Saito, M. Yoshino, Y. Yamazaki, and T. Namikawa, J. Mag. Soc. Jpn., 14, 309 (1990) (in Japanese).

27) T. Osaka, T. Homma, K. Kageyama, and Y. Matsunae, Denki Kagaku (presently Electrochemistry), 62, 987 (1994).
28) T. Osaka, T. Homma, K. Kageyama, Y. Matsunae, and O. Shinoura, J. Mag. Soc. Jpn., 18-S1, 183 (1994).

29) M. Takai, S. Takefusa, T. Yokoshima, and T. Osaka, J. Surf. Finish. Soc. Jpn., 48, 98 (1997) (in Japanese).

30) M. Takai, S. Takefusa, T. Yokoshima, and T. Osaka, Proc. of Fundamantal Aspects of Electrochemical Deposition and Dissolution Including Modeling, Electrochem. Soc. PV97-27, 358 (1997).

31) T. Yokoshima, H. Yuasa, M. Kim, and T. Osaka, J. Surf. Finish. Soc. Jpn., 49, 1336 (1998).

32) T. Yokoshima, D. Kaneko, T. Osaka, S. Takefusa, and M. Osaki, J. Magn. Soc. Jpn., 23, 1397 (1999) (in Japanese). 